\title{
SANEAMENTO E SOCIEDADE 5.0: INOVAÇÕES TECNOLÓGICAS PARA A REALIZAÇÃO DOS DIREITOS HUMANOS À ÁGUA E AO SANEAMENTO
}

\section{SANITATION AND SOCIETY 5.0: TECHNOLOGICAL INNOVATIONS FOR THE REALIZATION OF THE HUMAN RIGHTS TO WATER AND SANITATION}

\author{
Diego Augustus Senna ${ }^{(1)}$ \\ Doutorando e Mestre em Saneamento, Meio Ambiente e Recursos Hídricos (UFMG), MBA em Indústria 4.0 (PUC \\ Minas), Engenheiro Civil (UFMG). \\ Priscylla Pavlowa Senna ${ }^{(2)}$ \\ Graduanda em Arquitetura e Urbanismo (PUC Minas). \\ Luiz Philippe Pereira ${ }^{(3)}$ \\ Graduando em Ciência da Computação (UFMG). \\ Nathalia Roland ${ }^{(4)}$ \\ Doutora e Mestra em Saneamento, Meio Ambiente e Recursos Hídricos (UFMG), Engenheira Civil (UFMG). \\ Sonaly Rezende ${ }^{(5)}$ \\ Professora do Departamento de Engenharia Sanitária e Ambiental da UFMG, Pós-Doutora e Mestra em Saneamento, \\ Meio Ambiente e Recursos Hídricos (UFMG), Doutora pelo Centro de Desenvolvimento e Planejamento Regional da \\ UFMG (Cedeplar), Engenheira Civil (UFMG).
}

E-mail $^{(1)}$ : augustus.senna@yahoo.com.br

\section{RESUMO}

Serviços de saneamento são sociotécnicos. É necessário, portanto, considerar visão transdisciplinar, abordando o contexto externo sob as perspectivas ambiental, econômica e social. Atualmente, ocorre a transformação digital impulsionada pela Indústria 4.0, que resultou no desenvolvimento de conceitos como a Educação 4.0 e a Sociedade 5.0. Este artigo objetiva compreender as implicações desses conceitos para os Direitos Humanos à Água e ao Saneamento (DHAS) de forma a motivar a construção de políticas públicas mais abrangentes e inclusivas. Admite-se que as tecnologias podem ser colocadas a serviço da equidade, da participação social, da transparência e, principalmente, da educação. Abordagens como o EDUCAMENTO consideram mecanismos alternativos de comunicação, utilizando tecnologias de informação e conceitos como a gamificação, como caminhos para facilitar a realização dos DHAS. Dessa forma, a população pode ser incentivada por meio de linguagem atrativa e acessível, assimilando uma cultura sanitária mais sustentável e segura.

\begin{abstract}
Sanitation services are sociotechnical. It is, therefore, necessary to consider a transdisciplinary view, approaching the external context from the environmental, economic and social perspectives. Currently, the digital transformation is driven by Industry 4.0, which resulted in the development of concepts such as Education 4.0 and Society 5.0. This article aims to understand the implications of these concepts for the Human Rights to Water and Sanitation (HRWS) to motivate the construction of more comprehensive and inclusive public policies. It is admitted that technologies can be used at the service of equity, social participation, transparency and, above all, education. Approaches such as EDUCAMENTO consider alternative communication mechanisms, using information technologies and concepts such as gamification, as ways to facilitate the realization of HRWS. Thus, the population can be encouraged through attractive and accessible language, assimilating a more sustainable and safe sanitary culture.
\end{abstract}

Palavras-chave: Saneamento. Direitos Humanos à Água e ao Saneamento. Sociedade 5.0. Indústria 4.0. Educação 4.0. 


\section{INTRODUÇÃO}

O saneamento, além de constituir imprescindível mecanismo promotor da saúde, é elemento essencial para a construção da cidadania, pois estabelece base para a viabilização de outros direitos humanos. A falta de serviços de saneamento adequados, capazes de atender aos anseios da população, influencia diretamente nas condições de vida e na produtividade. Diante deste entendimento, um contexto de maior destaque internacional dado às ações de saneamento está se formando (HELLER et al., 2020). Há a oportunidade de serem estabelecidas interações mais proveitosas, voltadas à gestão do conhecimento, entre governos e distintos atores da sociedade civil.

Serviços de saneamento podem ser interpretados como sistemas sociotécnicos, uma vez que seu sucesso depende da interação entre a tecnologia, os responsáveis por implementá-la e os usuários. A análise sob a lente da sustentabilidade envolve, necessariamente, as esferas ambiental, econômica e social, e ganha especial relevância em contextos de limitação de recursos (ELKINGTON; ROWLANDS, 1999). Evidenciam-se, portanto, o aspecto transdisciplinar do saneamento e a importância de se considerar o contexto externo para a implementação de ações.

Atualmente, ocorre a transformação digital impulsionada pela Indústria 4.0, resultando no desenvolvimento de derivações, como a Educação 4.0. Visando um estado de plenitude social, surgiram formulações como a Sociedade 5.0. A relevância deste artigo reside na necessidade de compreender as implicações desses conceitos para os Direitos Humanos à Água e ao Saneamento (DHAS), motivando a busca por políticas públicas abrangentes, que abordem os temas simultaneamente. Objetiva-se, portanto, explorar esta correlação.

\section{DESENVOLVIMENTO}

O assunto encontra-se desenvolvido em três seções: i) 2.1, onde estão contextualizados princípios fundamentais dos DHAS; ii) 2.2, onde estão descritos os conceitos de Indústria 4.0, Educação 4.0, Sociedade 5.0 e correlações com os DHAS; e iii) 2.3, onde é apresentado o EDUCAMENTO.

\subsection{Contextualização e princípios dos DHAS}

O reconhecimento dos DHAS, em 2010 (ONU, 2010), amplia o conceito de acesso, desvinculandoo da mera presença ou não de uma ligação ou infraestrutura. De acordo com Albuquerque (2014), os DHAS são compostos por cinco critérios normativos:

1. Disponibilidade: instalações de abastecimento de água (AA) e de esgotamento sanitário (ES) devem atender às demandas presentes e futuras, estando disponíveis em ambientes onde é dedicada quantidade significativa de tempo. O AA deve ser suficiente e contínuo. A coleta, o transporte, o tratamento e a disposição final dos esgotos devem ser assegurados.

2. Qualidade e segurança: a água deve ser isenta de microrganismos e substâncias químicas ou radioativas que provoquem riscos à saúde humana. São previstos ambientes limpos, bem cuidados, com disponibilidade de água e sabão, adequados aos diversos tipos de higiene.

3. Acessibilidade física: as infraestruturas devem ser de fácil acesso, considerando-se demandas específicas relacionadas às limitações de mobilidade de crianças, idosos e pessoas com deficiências. $\mathrm{O}(\mathrm{s})$ ponto(s) de água e o banheiro devem estar dentro ou nas imediações de cada local requerido.

4. Acessibilidade financeira: as tarifas de AA e ES não devem limitar a capacidade de adquirir outros serviços e bens relativos aos demais direitos humanos (alimentação, moradia, saúde e educação). Os Estados têm a obrigação de criar subsídios para os mais pobres.

5. Aceitabilidade: as soluções podem ser abandonadas caso não correspondam aos padrões 
sociais e culturais de seus usuários. As infraestruturas devem ser consideradas aceitáveis e a água deve ter cor, gosto e odor admitidos como adequados. Deve-se garantir a privacidade e especificidades relacionadas com o gênero, com especial atenção às mulheres e meninas.

Além do conteúdo normativo dos DHAS, os princípios gerais dos direitos humanos também devem ser rigorosamente observados: igualdade e não-discriminação, acesso à informação e transparência, participação social, prestação de contas, sustentabilidade, realização progressiva e não-retrocesso (ALBUQUERQUE, 2014).

Nas últimas décadas, esforços globais têm sido realizados para promover melhorias no acesso ao saneamento, destacando-se os Objetivos do Desenvolvimento do Milênio (ODM) - encerrados em 2015 - e os atuais Objetivos do Desenvolvimento Sustentável (ODS), parte da Agenda 2030. Considerável parcela da população mundial ainda sofre com a exclusão sanitária e a injustiça social. Os impactos são sentidos especialmente por populações rurais, moradores de favelas urbanas, mulheres e crianças (WHO/UNICEF, 2012). O enquadramento dos DHAS consiste em marco importante, uma vez que influencia a lógica de se planejar, monitorar e avaliar as políticas para o setor, evidenciando populações historicamente marginalizadas, especialmente em países em desenvolvimento, por vezes ainda distantes da universalização dos serviços.

\subsection{Correlações entre a Indústria 4.0, a Educação 4.0, a Sociedade 5.0 e os DHAS}

A “Plattform Industrie 4.0”, lançada pelo governo alemão, em 2013, pode ser definida como novo paradigma industrial que organiza, dentro de um mesmo contexto, as diversas tecnologias provenientes da digitalização, com destaque para aquelas referentes à informação e à comunicação - TICs (KAGERMANN et al., 2016). Procura-se, dentre outros fatores: i) o direcionamento do crescimento da automação e da circulação de dados; ii) o aumento das eficiências econômica e ecológica; iii) a redução do tempo necessário para a pesquisa e o desenvolvimento de inovações; e iv) a adaptação às necessidades das pessoas (LASI et al., 2014). A Quarta Revolução Industrial diferencia-se das anteriores por adquirir formato mais aproximado de conjunto de políticas públicas.

As novas competências exigidas pela Indústria 4.0 atuam como estímulos para a modificação de sistemas educacionais tradicionais. A Educação 4.0, elaborada neste contexto, baseia-se na personalização do aprendizado, sendo o foco direcionado à experiência pessoal de cada indivíduo. As formas de apresentação do conhecimento, considerando as particularidades do público, tornaram-se tão importantes quanto o próprio conteúdo exposto (ALMEIDA; SIMOES, 2019).

A transformação digital pode propiciar inúmeros benefícios, mas existe preocupação quanto à prevalência de uma visão tecnocêntrica. Em 2016, a base proposta para a Indústria 4.0 foi utilizada pelo governo japonês para a definição de um novo conceito, a Sociedade 5.0, com diretivas orientadas para a prosperidade social. Segundo Potocan, Mulej e Nedelko (2020), há alinhamento aos ODS da ONU, tomando as tecnologias como caminho para a resolução de problemas sociais e a busca por sustentabilidade. A Federação Empresarial do Japão (2017, p. 8), estabelece que "tecnologias digitais e dados sejam utilizados para criar uma sociedade onde as pessoas liderem diversos estilos de vida e busquem a felicidade de suas próprias maneiras".

Os DHAS estão relacionados com a Indústria 4.0, primeiramente, por meio da disponibilização de base tecnológica. Há o desenvolvimento acelerado de soluções mais eficientes, o que pode resultar em redução de custo - contribuindo com o investimento em ações inclusivas - e ganho ambiental. É natural, portanto, que os responsáveis pela formulação de políticas públicas devam estar atentos às inovações. Outro ponto baseia-se na forte presença de TICs: há o estímulo à participação social através do acesso facilitado à informação, diante da popularização cada vez mais acelerada de smartphones e infraestrutura de rede, o que contribui para a transparência e a prestação de contas. 
A flexibilização do aprendizado, decorrente da Educação 4.0, promove a construção de uma cultura sanitária por meio do estímulo ao reconhecimento, pelos próprios usuários, de seus direitos e deveres no contexto de ações de saneamento, diante da identificação dos cinco critérios normativos essenciais e da obrigação do Estado em fornecer serviços universais, valorizando a equidade e a não-discriminação das populações mais vulneráveis. Neste contexto, há direta conexão com a Sociedade 5.0, fortemente ligada aos ODS e aos DHAS, uma vez que é reconhecida a necessidade de avaliação das especificidades de cada população para o alcance da prosperidade social, considerando a expansão de forma progressiva, com respeito ao modo de vida de cada indivíduo, que precisa se sentir confortável com as mudanças propostas e realizadas. É também prevista a compulsoriedade de subsídios, evitando que as políticas públicas estejam submetidas a retrocessos.

\subsection{EDUCAMENTO: educação para o saneamento no contexto digital}

Para que o acesso ao AA e ao ES seja democratizado, garantindo a realização dos DHAS, é importante que existam instrumentos de comunicação e de transmissão de informações. $O$ EDUCAMENTO se apoia na educação que emerge da realidade experimentada a partir de conhecimento prévio, que tem forte relação com a cultura ancestral, mas também é permeada pela cultura de massa. O ponto de partida é o conceito de educação popular, de Paulo Freire. As ferramentas de interação e aprendizagem transportam usuários, gestores e políticos para novos mundos, através da sensibilização, permitindo que a discussão sobre as demandas e disposições para a oferta de serviços de saneamento seja dinâmica e potencialize a disseminação de práticas mais sustentáveis, capazes de orientar conceitos, regras e modos de vida - nos planos individual e coletivo - a seguirem caminhos seguros. Segundo Ribeiro et al. (2017), a comunicação permite o compartilhamento de ideias, valores e crenças, promovendo a assimilação de narrativas que conformam visões sanitárias, importantes para delinear estratégias.

A educação, segundo Paulo Freire, é um processo de formação essencial à sobrevivência humana, cuja duração se confunde com a da própria vida (FREIRE, 2000). Não há neutralidade da educação, seja ela permanente ou não. Diante de comportamentos socioculturais distintos, educação e aprendizagem são conceitos ambíguos. Assim como o saneamento, a educação é um direito humano capaz de potencializar a realização de outros direitos.

O uso de metodologias alternativas para a solução de problemas cotidianos tem sido ampliado na área de saneamento, tendo em vista o destaque alcançado pelo eixo estratégico educação e participação social na formulação do Programa Nacional de Saneamento Rural (PNSR) e na elaboração de planos municipais de saneamento, em curso no País. No PNSR (BRASIL, 2019), o tema da educação compreendeu as instâncias públicas e privadas, sendo o conhecimento construído conforme três processos delineados por Gohn (2006): i) os formais, segundo normas escolares e conteúdos sistematizados que visam, entre outros fins, a formação de "cidadãos ativos"; ii) os não formais, complementares à formação oficial ou profissionalizante, que formam os "cidadãos do mundo", que absorvem e disseminam a cultura de massa; e iii) os informais, que ocorrem cotidianamente, fundados na convivência e compartilhamento.

Mészaros (2002) mobiliza a prática educacional na perspectiva da "sociabilidade livre da alienação", como um vetor de reprodução de novos conceitos e novas formas de se enxergar o mundo, na contramão da hegemonia do capital, repleta de contradições que perpetuam a reprodução das desigualdades socioeconômicas.

O planejamento de cidades inteligentes é alvo de críticos à priorização de soluções lideradas pelo mercado e reprodução do capitalismo neoliberal, onde a estrutura prevalente é tecnocrática, implementada de cima para baixo e, por isso, respondendo pela perpetuação das desigualdades sociais (CARDULLO; KITCHIN, 2019). Visando à construção de caminho paralelo a este, no qual 
a comunicação e a construção de conhecimento são mecanismos promotores da realização dos DHAS, o EDUCAMENTO reforça a necessidade de serem criadas novas abordagens de mobilização social e de ativismo popular e comunitário.

O EDUCAMENTO envolve, pois, ferramentas para mobilizar a participação de usuários e propiciar o seu envolvimento com o poder público e os gestores técnicos, em uma conjugação de esforços para alcançar a universalização, com equidade. Na perspectiva dos DHAS, o EDUCAMENTO enfrenta os desafios apoiando-se em tecnologias como a inteligência artificial e conceitos inovadores, bem como o monitoramento, em tempo real, da salubridade ambiental, em processos alinhados a princípios de sustentabilidade e sociotécnica (COSGROVE; LOUCKS, 2015).

A transformação digital viabiliza mais do que nunca a comunicação com os usuários de serviços de saneamento. De acordo com a Pesquisa Nacional por Amostra de Domicílios (PNAD) Contínua de 2019, do Instituto Brasileiro de Geografia e Estatística (IBGE, 2021), o acesso à Internet está presente em $82,7 \%$ dos domicílios brasileiros, ao passo que $81,0 \%$ da população maior de 10 anos tem aparelho celular pessoal. Portanto, as alternativas tecnológicas trazem grande alcance para o diálogo sobre os temas do EDUCAMENTO. Abordagens como a gamificação, conteúdos interativos em vídeo, websites e portais de fácil acesso apresentam-se cada vez mais como facilitadores da assimilação de conteúdo e promotores da sensibilização. Conforme o contato com esses tipos de tecnologias se torna cada vez mais democrático e inclusivo, mais ferramentas são desenvolvidas. Plataformas expositivas como YouTube e Medium facilitam a divulgação de informações, enquanto plataformas interativas como Kahoot possibilitam que qualquer usuário ou organização desenvolva conteúdos gamificados com alto potencial de impacto.

\section{CONSIDERAÇÕES FINAIS}

As mudanças necessárias à ampliação do acesso ao AA e ES e, por conseguinte, ao alcance dos DHAS, passam por assimilações provenientes de processos educacionais formais, não formais e informais. A escola introduz aspectos formais visando à formação, em um dado patamar, de sujeitos ativos e conscientes; a cultura e o conhecimento ancestral estão enraizados na transmissão de conhecimento no âmbito familiar e de vizinhança; em outros contextos, os não formais, há o fomento à assimilação a partir de didáticas particularmente indutoras de alguma especialização. Todas essas vertentes são permeadas pela cultura de massa, facilmente assimilada e difundida.

Tendo em conta a importância de fomentar a realização dos DHAS, é relevante repercutir o conceito de Saneamento 5.0, cunhado a partir de compreensões que residem na Sociedade 5.0, na Indústria 4.0 e na Educação 4.0, para que a comunicação alcançada por meio de instrumentos baseados em TICs ocorra visando também à democratização do acesso ao saneamento. Além disso, dada a proximidade entre a Sociedade 5.0, os ODS e os DHAS, considera-se que as políticas públicas devem desenvolver esses conceitos em conjunto, e não apenas em paralelo, para potencializar os impactos e o alcance das ações.

\section{AGRADECIMENTOS}

Os autores agradecem à CAPES, pelo fornecimento de bolsa de mestrado, e ao CNPq, pelo fornecimento de bolsas de produtividade em pesquisa e de iniciação científica.

\section{REFERÊNCIAS}

ALBUQUERQUE, C. Realising the human rights to water and sanitation: a handbook by the UN special rapporteur Catarina de Albuquerque. Portugal: Human Rights to Water \& 
Sanitation, 2014.

ALMEIDA, F.; SIMOES, J. The Role of Serious Games, Gamification and Industry 4.0 Tools in the Education 4.0 Paradigm. Contemporary Educational Technology, v. 10, n. 2, p. 120-136, 2019. DOI: https://doi.org/10.30935/cet.554469.

CARDULLO, P.; KITCHIN, R. Smart urbanism and smart citizenship: The neoliberal logic of 'citizen-focused' smart cities in Europe. Environment and Planning C: Politics and Space, v. 37, n. 5, p. 813-830, 2019. DOI: https://doi.org/10.1177/0263774X18806508.

COSGROVE, W. J.; LOUCKS, D. P. Water management: Current and future challenges and research directions. Water Resources Research, v. 51, n. 6, p. 4823-4839, 2015. DOI: https://doi.org/10.1002/2014WR016869.

ELKINGTON, J.; ROWLANDS, I. H. Cannibals with forks: the triple bottom line of 21st century business. Alternatives Journal, v. 25, n. 4, p. 42-43, 1999.

FEDERAÇÃO EMPRESARIAL DO JAPÃO (KEIDANREN). Society 5.0: Co-creating the future. 2017. Disponível em: https://bit.ly/3akkpum. Acesso: 10 mai. 2020.

FREIRE, P. Ideologia e educação: reflexão sobre a não neutralidade da educação. In: GADOTTI, M. A educação contra a educação: o esquecimento da educação através da educação permanente. Rio de Janeiro: Paz e Terra, p. 15-19, 1981.

GOHN, M. G. Educação não formal e o educador social. Atuação no desenvolvimento de projetos sociais. São Paulo: Cortez, 2010. 104p.

HELLER, L. et al. Overview of 12 Years of Special Rapporteurs on the Human Rights to Water and Sanitation: Looking Forward to Future Challenges. Water, v. 12, n. 9, 22 p., 2020. DOI: https://doi.org/10.3390/w12092598.

INSTITUTO BRASILEIRO DE GEOGRAFIA E ESTATÍSTICA (IBGE). Acesso à internet e à televisão e posse de telefone móvel celular para uso pessoal 2019 / IBGE, Coordenação de Trabalho e Rendimento. Rio de Janeiro: IBGE, 2021. Disponível em: https://bit.ly/3vmFWvS. Acesso: 17 out. 2021.

KAGERMANN, H. et al. (Eds). Industrie 4.0 in a Global Context: Strategies for Cooperating with International Partners (acatech STUDY). Munique: Herbert Utz Verlag, 2016. Disponível em: https://bit.ly/3AjjqFb. Acesso: 20 mai. 2021.

LASI, H. et al. Industry 4.0. Business and Information Systems Engineering, v. 6, n. 4, p. 239242, 2014. DOI: https://doi.org/10.1007/s12599-014-0334-4.

MÉSZÁROS, I. A educação para além do capital. São Paulo: Biotempo, 2002.

ONU. General Assembly Resolution A/RES/64/292. The human right to safe drink water and sanitation. New York, 2010. Disponível em: https://bit.ly/3FrHsBK. Acesso: set. 2021.

POTOCAN, V.; MULEJ, M.; NEDELKO, Z. Society 5.0: balancing of Industry 4.0, economic advancement and social problems. Kybernetes, v. 50, n. 3, p. 794-811, 2020. DOI: https://doi.org/10.1108/K-12-2019-0858.

RIBEIRO, M. G.; FARIA, P. A.; DIAS, A. P. Educação e Tecnologia Social. In: GONDIM, G. M. M.; CHRISTÓFARO, M. A. C.; MIYASHIRO, G. M. (Org.). Técnico em Vigilância em Saúde Fundamentos. Vol. 2. Rio de Janeiro: EPSJV, 272 p., 2017. Disponível em: https://bit.ly/3AmSNiw. Acesso: 4 out. 2021.

WHO/UNICEF. Water, sanitation, hygiene. Key terms. WHO/UNICEF Joint Monitoring Report 2012, 2012. Disponível em: https://bit.ly/3BlRWjy. Acesso: mar. 2020. 